\title{
Human Sensing in Reverberant Environments: RF-based Occupancy and Fall Detection in Ships
}

\author{
Marwan Yusuf, Emmeric Tanghe, Brecht De Beelde, Pierre Laly, Matteo Ridolfi, Eli De Poorter, Luc Martens, \\ Davy P. Gaillot, Martine Lienard, and Wout Joseph
}

\begin{abstract}
In this paper, we explore the possibility of estimating the number of people inside a below-deck ship compartment using the theory of room electromagnetics. Most RF-based occupancy detection solutions focus on residential environments. Confined metallic spaces such in industrial environments are more hostile and exhibit features of a reverberant cavity. The theory of room electromagnetics provides a simple characterization of microwave propagation in such environments. By considering the indoor environment as a lossy cavity, the exponential decay rate of the power-delay profile (PDP), also known as reverberation time (RT), is related to the total absorption inside the room. First, we verify the reverberating nature of the room by measuring the RT at different locations inside the room. The PDPs are calculated from the channel impulse response (CIR) measured using the MIMOSA radio channel sounder. Then, the relation between RT and the number of people inside the room is investigated. We show that it is possible to estimate the number of stationary people with a good accuracy, depending on the number of antennas used. With a success rate of $88 \%$, the estimation error is only one person when 16 spatially averaged PDPs are used. Higher success rates are achievable with more spatial averaging. Moreover, off-the-shelf (OTS) ultra wide band (UWB) devices are used to estimate the number of people inside the same room. Results show that temporal averaging of PDPs can further improve the success rate to $95 \%$ when people inside the room are moving. In addition, the detection of a lone person falling to the ground is investigated based on the Doppler analysis of the measured CIRs. It is shown that the Doppler spread in case of a fall has higher peak values compared to normal activities, such as walking and sitting. With the use of a simple Bayes classifier, a fall is detected with $98 \%$ accuracy and $100 \%$ sensitivity.
\end{abstract}

Index Terms-ships, propagation measurements, room electronmagnetics, reverberation time, human sensing, counting people, indoor occupancy, fall detection, Doppler

\section{INTRODUCTION}

A $\mathrm{N}$ increasingly common requirement of smart systems is to extract information about the people present in an environment in a device-free way, meaning that humans are not

Copyright (c) 2021 IEEE. Personal use of this material is permitted. However, permission to use this material for any other purposes must be obtained from the IEEE by sending a request to pubs-permissions@ieee.org.

This work is part of the Internet-of-Shipping (IoS) research project. IoS is co-funded by IMEC and Flanders Innovation \& Entrepreneurship (VLAIO) Authors would like to thank Ivan Renette (Exmar ship management), Ben Van Herbruggen, Nicola Macoir (IDLab-UGent), and Abdil Kaya (UAntwerp) for facilitating the measurement campaign.

Marwan Yusuf, Brecht De Beelde, Emmeric Tanghe, Matteo Ridolfi, El De Poorter, Luc Martens and Wout Joseph are with the Department of Information Technology, Ghent University-IMEC, Belgium (e-mail: marwan.yusuf@ugent.be)

Pierre Laly, Davy P. Gaillot and Martine Lienard are with the Electronics Department, IEMN-TELICE, University of Lille, France.

Manuscript received XXX, XX, 2019; revised XXX, XX, 2019. expected to carry any dedicated devices or passive tags. Basic human-sensing such as people counting is of high importance in the context of Internet-of-Things, driving many applications: from building automation to surveillance and safety monitoring in case of natural or man-made disasters. Hence, deploying wireless networks in confined, reflective spaces such as found in metallic warehouses, aircraft cabins, and belowdeck compartments in ships is essential to the envisioned Industry 4.0 and intelligent transportation systems (ITS). In particular, below-deck spaces in ships have been a primary focus of a number of studies, exploring the wireless radio frequency $(\mathrm{RF})$ propagation and communication performance [1]-[4]. Current shipboard monitoring systems use extensive lengths of cables to connect a massive number of sensors to control units. Wired installation during ships construction results in a high cost and weight. In addition, ships represent a harsh environment wherein wires are vulnerable to moisture, heat and hazardous elements, making maintenance a very difficult task. On the other hand, wireless communication is a serious challenge in such hostile environments [4]. These highly reflective environments are characterized by rich electromagnetic scattering, time and angular dispersion, and can exhibit features of a complex reverberant cavity. While typical indoor environments (e.g., residential and office) show RMS delay spreads of $15-100 \mathrm{~ns}$ at $2.4 \mathrm{GHz}$, highly reverberant environments can reach up to $1200 \mathrm{~ns}$ [5].

With regard to human counting or occupancy detection, different types of sensing solutions are available today, each having its advantages as well as limitations [6]. Solutions vary in the targeting approach (e.g. counting people through doorways or within certain areas) or the sensing modality (visual, thermal, etc.). Image-based solutions, for example, are prone to blind spots, sensitive to environmental condition like lighting, smoke etc., pose privacy issues and are computationally expensive due to the image processing, which is often based on machine learning algorithms. On the other hand, RFbased solutions have a great potential in overcoming many of those drawbacks [6]. Using RF signals such as Wi-Fi or Zigbee has the benefit of exploiting the deployed networks for sensing as well, without additional infrastructure. RF sensing is mainly based on received signal strength indication (RSSI) or channel state information (CSI). This limits the accuracy of such methods in hostile propagation environments, even with the use of probabilistic and machine learning algorithms [6]. Another limitation is that they usually require extensive training, which must be performed in the same targeted environment and for each number of the supported count range. 
Another RF-based solution makes use of the ultra wide band (UWB) technology. UWB is a technology that can be used for both communication and sensing. The IEEE 802.15.4a is the international standard defining the UWB physical and MAC layers. This standard is designed to deliver very accurate measurements of the Time of Flight ( $\mathrm{ToF}$ ) of the radio signal, leading to localization with centimeter accuracy. Moreover, it provides simultaneous two-way communications up to 27 Mbps and consumes low energy, making it a perfect fit for battery-powered devices [7]. In 2018, further security aspects are added to the physical layer, forming the $802.15 .4 \mathrm{z}$ standard. On the other hand, UWB sensing is based on multi-target detection via radar transceivers [6]. An impulse radio (IR) UWB radar transmits a narrow impulse signal that occupies a wide bandwidth in the frequency domain, with fine resolution and high penetration. The radar then receives and analyses the backscattered signal to infer the number of people and distances within the radar range. While it has the best performace among the RF solutions, its need for a dedicated radar sensor adds to the mentioned limitations [6].

A sensing solution that performs well in certain indoor scenarios, like in a lab or office environment, can not guarantee the same performance in industrial environments. The authors in [8], for example, used the IR UWB radar for people counting in two environments: an indoor room and an elevator with metal structure, and noticed a performance degradation in the elevator compared to the room. As aforementioned, highly reflective environments cause rich multipath scattering. Reflections from targeted humans can easily be buried in the scattered multipath components that can contribute up to $95 \%$ of the total power density [9]. Nonetheless, the method proposed in this paper exploits the diffuse multipath scattering in such reflective environment to estimate the number of people indoors. The main difference is that, as the environment becomes more reverberant, our techniques performance improves, while other RF techniques performance deteriorates.

Similar to Room Acoustics [10], Room Electromagnetics theory views the indoor environment as a lossy cavity, where all the effective losses can be described by the exponentially decaying tail of the power-delay profile (PDP) [11] shown in Fig. 1. The decay time constant, also known as the reverberation time (RT), is a function of the volume and the absorption area of the room. Several studies were conducted based on this theory, e.g. [12]-[14]. They validate the use of the acoustic reverberation models in electromagnetics, and show that the reverberation is confined in the room where the transmitter $(\mathrm{Tx})$ and receiver $(\mathrm{Rx})$ are located, and that the RT is location and antenna independent. The RT has previously been used to calculate the effective absorption coefficient as a single parameter that characterizes a room [11]. In addition, the mean received diffuse power can be determined everywhere by knowing RT and the volume of the room [12]. From the assumption of diffuse scattering, the RT allows the determination of the path loss and delay time parameters [13]. Specific absorption rate (SAR) as a basic restriction for RF human exposure is often found numerically from knowledge of the distribution of complex permittivity in the body, and experimental values are scarce. A measurement-

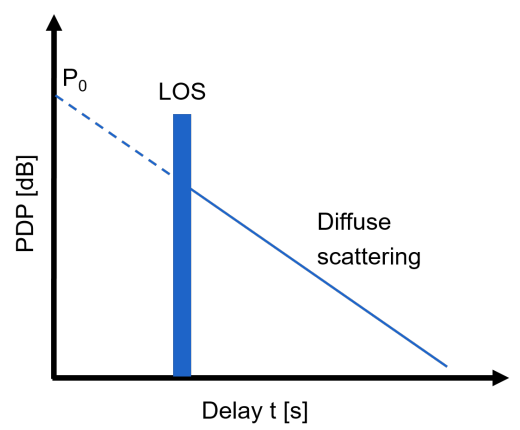

Fig. 1. Theoretical model of propagation including a LOS and an exponential tail with time constant $\tau$

based approach based on the RT is a suitable alternative for the assessment of the whole-body averaged SAR [14].

Room Electromagnetics relates the RT to the absorption inside the room. The presence of people in the room will alter the total absorption area, and hence, the RT of the room. The inverse relation between the number of people and the RT has been recorded in reverberation chambers using human phantoms [15] and inside cars with real humans [16]. In this work, we exploit this relation to detect the presence and number of people inside ship compartments. This should work in the whole room where the reverberating field fills up. Wherever the person is in the room (e.g. behind a pipe), he will perturb the reverberating field, and this will show up in the RT. The PDP is calculated based on the channel impulse response (CIR), a quantity most wideband communication systems measure via e.g. pilot symbols [17]. This gives our solution the capability to be integrated in communication networks such as UWB systems [7]. To this end, the CIR is measured in a below-deck room and the RT is calculated by means of linear regression. With a changing number of people in the room, the number of people in each case is estimated using the theory of Room Electromagnetics.

In addition, operation onboard ships for example sometimes requires crew members to work alone in isolated rooms. With the lack of reliable communication below-deck, a person falling on the ground can become a serious situation if not detected in time. A complementary feature that detects if a person alone has fallen from a standing position is highly desirable. RF-based fall detection in the literature is mainly based on Doppler radar sensors that scan the environment at rates higher than $1 \mathrm{KHz}$ [18]. Other solutions that use Wi-Fi signals are also available [19], [20]. They use machine learning by extracting features from the CSI that are unique for the fall compared to other normal activities. However, all these studies focus on residential environments, which are far less hostile than industrial ones. Hence, this work explores the possibility of fall detection in harsh environments using Doppler analysis of the CIRs available in wideband communication systems at a much lower rate than radar solutions. The contributions of this paper can then be summarized as:

- Investigating the reverberating nature of confined metallic environments such as found in ships

- Design of an algorithm for device-free occupancy detec- 

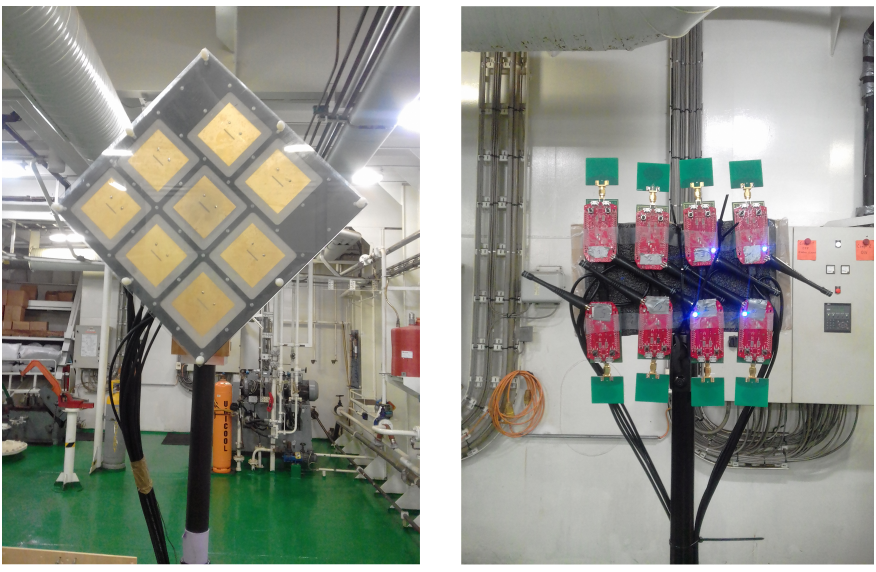

Fig. 2. The rectangular antenna array of the channel sounder (left) and the equivalent UWB nodes array (right)

tion in reverberant environments based on Room Electromagnetics

- The algorithm uses CIRs and thus can be integrated in wideband communication systems

- Experimental validation of the algorithm in a realistic ship environment using a radio channel sounder as well as OTS hardware

- Exploring the possibility of fall detection in reverberant environments based on the CIRs

The outline of this paper is as follows. The measurement setup and scenario are described in Section II. Section III describes the methodology to extract the RT, while in Section IV processing of the measurement data is presented. Section $\mathrm{V}$ shows the estimation results for the number of people, while the fall detection is discussed in Section VI. Finally, conclusions are drawn in Section VII.

\section{Measurement Configuration and Scenario}

Channel measurements are first performed with the MIMOSA radio channel sounder [21]. The multidimensional polarimetric CIR is measured in the spatial and polarization dimensions to investigate the reverberant behavior of the room. It is then used to test the occupancy detection algorithm when the people are standing still as a proof-of-concept. The next step is to verify the algorithm performance with OTS products that provide the CIR. Hence, UWB devices are used for the occupancy detection when the people move in the same room, which is a more realistic scenario. This section includes the details of the measurement configurations and scenario.

\section{A. MIMOSA Channel Sounder}

The sounder operates with a carrier frequency of $1.35 \mathrm{GHz}$ and the transmission bandwidth is $80 \mathrm{MHz}$. It uses dualpolarized patch antenna arrays with horizontal $(\mathrm{H})$ and vertical (V) polarization. For this measurement campaign, 8-element rectangular arrays are used at both $\mathrm{Tx}$ and $\mathrm{Rx}$ as shown in Fig. 2 (left). Orthogonal frequency division multiplexing is used to encode 8 parallel transmit channels, and by connecting each to a two-port RF switch for the two polarizations, a

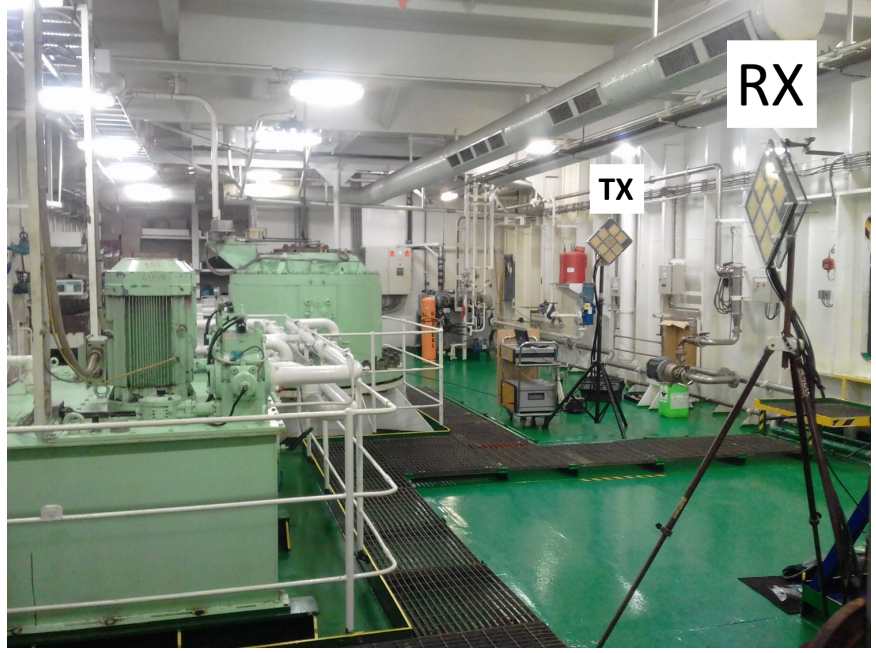

Fig. 3. Below-deck ship compartment with rectangular antenna arrays of the channel sounder

TABLE I

MIMOSA Channel SOUNDER SPECIFICATIONS

\begin{tabular}{lr}
\hline Parameter & Setting \\
\hline center frequency & $1.35 \mathrm{GHz}$ \\
bandwidth & $80 \mathrm{MHz}$ \\
number of Tx and Rx antennas & 8 \\
Tx and Rx polarization & $\mathrm{H} / \mathrm{V}$ \\
number of OFDM subcarriers & 6560 \\
OFDM symbol duration $T_{S}$ & $81.92 \mu \mathrm{s}$ \\
cyclic prefix duration $T_{C P}$ & $0 \leq T_{C P} \leq T_{S}$ \\
$16 \times 16$ channel acquisition time & $2\left(T_{S}+T_{C P}\right) \leq 327.68 \mu \mathrm{s}$ \\
\hline
\end{tabular}

total of $16 \times 16$ channels are measured. The channel sounder is fully parallel: the data from each transmit antenna are simultaneously modulated onto the carriers using interleaved frequency division multiple access. Table I summarizes the technical specifications of MIMOSA [21].

\section{B. OTS Hardware: UWB Devices}

UWB is one of the most promising technologies for realtime location systems (RTLS) indoors due to its accurate positioning capabilities, immunity against multipath fading, and excellent resilience against narrowband interference [7]. In our experiment, we use a newly developed open source hardware platform based on Decawave's DW1000 UWB transceiver chips with support of both long-range sub- $\mathrm{GHz}$ and $2.4 \mathrm{GHz}$ back-end communication between nodes [22]. An external omni-directional antenna with vertical polarization is connected to each node. To calculate the distance between 2 nodes based on the ToF, asymmetric two-way ranging protocol [23] is used at a rate of $25 \mathrm{~Hz}$. One of the optional outcomes of this protocol is the CIR estimate of size equal to 1016 samples. Hence, the CIRs are recorded with $900 \mathrm{MHz}$ bandwidth around $4 \mathrm{GHz}$ center frequency using channel 4 of the DW1000 chip [23].

\section{Measurement Scenario}

The measurement campaign is carried out in the steering gears room of a bulk carrier vessel, shown in Fig. 3. The Tx 
and Rx of the channel sounder are placed inside the room and the door is kept closed during the measurement. The room has a height of $4 \mathrm{~m}$ and a floor area of $150 \mathrm{~m}^{2}$, approximately. The $\mathrm{Rx}$ antenna array is fixed at $2 \mathrm{~m}$ height, pointed towards the corner of the room [1]. The reverberating nature of the room is first investigated by comparing the RT at random locations inside the room. This is done by placing the Tx antenna array at 6 different locations to measure the CIRs. Then, the Tx is fixed and the CIRs are recorded for different numbers of people inside the room, ranging from 0 to 6 persons standing still at random locations. The channel in each case is measured 200 times in static conditions and averaged to reduce measurement noise. Hence, a total of $7 \times 64 \times 4$ PDPs are calculated, where the value 64 results from the $8 \times 8$ antenna elements, and 4 from the TxRx polarization combinations (VV, $\mathrm{HV}, \mathrm{VH}, \mathrm{HH})$. The same scenario is repeated using the UWB nodes with the difference that the people are allowed to move inside the room. A setup of $8 \times 8 \mathrm{UWB}$ nodes is used to measure the 64 spatially uncorrelated CIRs 200 times for each number of people. The Rx antennas setup of both measurements is shown in Fig. 2.

\section{Methodology}

In order to estimate the number of people inside the room, we first need to extract the RT. In indoor propagation, multiple reflections and scattering lead to an exponential decay of power with a decay constant $\tau$ representing the RT. The diffuse scattering model in Fig. 1 can be written as

$$
P(t)=P(0) \exp (-t / \tau)
$$

where $t$ is the time of arrival on the delay axis and $P(t)$ is the corresponding received power. According to Room Electromagnetics, the RT can be expressed as [11]

$$
\tau=\frac{4 V}{c A_{n}}
$$

where $V$ is the room volume, $A_{n}$ is the total absorption area and $c$ is the velocity of light. In a fixed environment, where the contents of the room do not change except for the number of people inside, the total absorption area can be written as

$$
A_{n}=A_{0}+n \times A C S
$$

where $A_{0}$ is the absorption area of the room without people, $n$ is the number of people in the room and $A C S$ is the whole-body absorption cross-section area of the human body [15]. Each additional person in the room increases the total absorption area by the amount of the $A C S$ of the human body. The PDP is calculated as

$$
\mathrm{PDP}=\frac{1}{N} \sum_{N}|\mathrm{CIR}|^{2}
$$

where $\mathrm{N}$ is the number of CIRs aligned to the same LOS delay as in [24]. Once the RT is extracted from the PDP, it is possible to assess the absorption area $A_{n}$ from (2). Assuming the $A C S$ is known, the number of people $n$ can then be calculated from (3).

For the extraction of the RT, the slope of the PDP exponential decay needs to be calculated. However, the tail of the

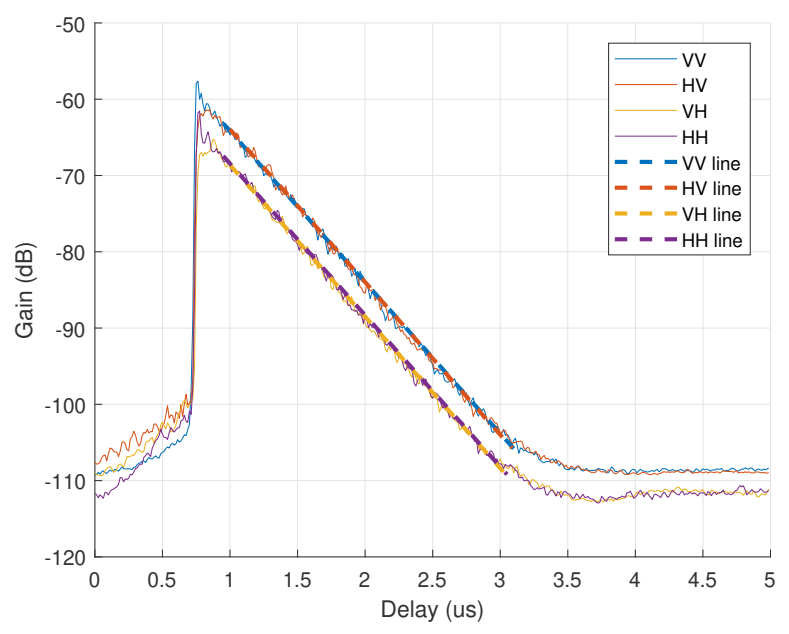

Fig. 4. PDP and linear regression for different polarizations in case of an empty room

PDP is not perfectly linear due to noise, LOS and specular components. A least squares regression line is thus used to fit the PDP over a delay range, and the choice of this range is done automatically. First, to avoid LOS, the starting point is taken as the mean arrival time $T_{m}$ given by

$$
T_{m}=\frac{\sum t P(t)}{\sum P(t)}
$$

Second, the noise level is calculated by averaging the power values at large delays from the PDP where no multipath components above the noise are expected. Finally, in order to avoid the noise floor, the ending point of the delay range is taken when the power level reaches $5 \mathrm{~dB}$ above the calculated noise level.

\section{Experimental Results And Preprocessing}

\section{A. RT Extraction}

In order to investigate the reverberating nature of the environment, the RT is calculated at 6 different locations in case of an empty room. The PDP per location is measured using the channel sounder and spatially averaged over all the antenna elements before extracting the RT, to remove smallscale fading. Fig. 4 shows the PDP at one location for different polarizations. The figure shows the identical diffuse power level of the co-polar and cross-polar channels, suggesting that the polarization states in the room are uniformly distributed (XPD of one), as is expected in a reverberating environment. To further validate the assumption of reverberating fields, the RT is calculated at 6 random locations in the room. Fig. 5 shows the RT at each location for different polarizations. It is clear that the RT value is independent of the location as well as the polarization of the antennas, with a relative standard deviation of $0.99 \%$. This verifies the reverberating nature of the room, in accordance with [14].

With the Tx fixed, the RT is calculated when the room is occupied by people, starting from 1 up to 6 persons. Fig. 6 shows the RT versus the number of people for different 


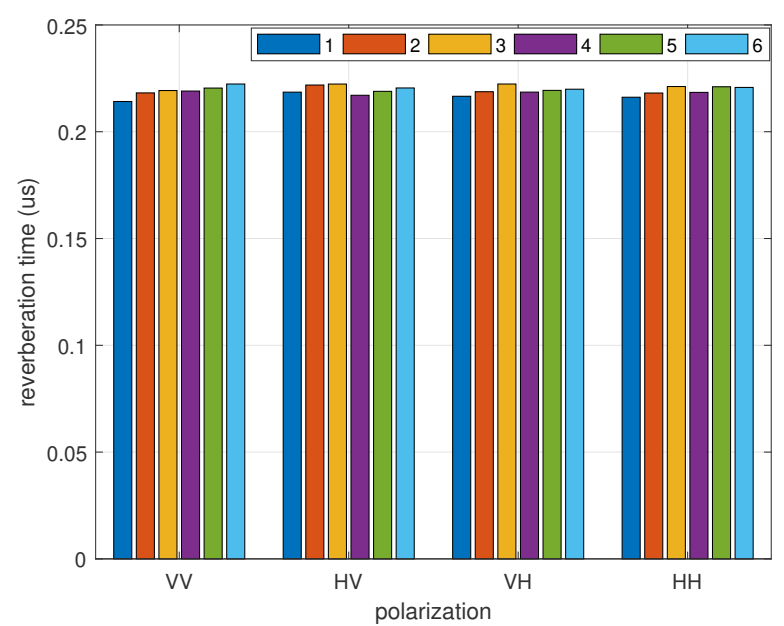

Fig. 5. RT at 6 locations for different TxRx polarization combinations in case of an empty room

polarizations. The figure clearly indicates that the RT is inversely proportional to the number of people. This validates the relation between the RT, absorption area, and the number of people in the room for different polarizations. Fig. 6 also shows that the RT for VV is slightly lower than the average of all polarizations, suggesting that the human body is more prone to absorbing vertically polarized waves. Nevertheless, the RT values for different polarizations are aggregated to form a larger sample size to be used for the occupancy detection in the next section, as they are similar enough with relative standard deviation of $0.61 \%$.

\section{B. Absorption Area and Calibration}

Since the average $A C S$ of the people inside the room is assumed to be known, it would be of interest to first calculate the $A C S$ based on our measurements and compare it to prior studies. From (3), the $A C S$ is the slope of the linear regression of different absorption areas $A_{n}$ related to different numbers of people $(n=0,1, . ., 6)$. The calculation of $A_{n}$ is given in (2) by averaging the PDPs of all polarizations before extracting the RT. This gives an $A C S$ of $1.3 \mathrm{~m}^{2}$ based on our experiment. The whole-body $A C S$ was measured in a reverberation chamber in [15] and was found to be 11.01$1.11 \mathrm{~m}^{2}$ for a $1.73 \mathrm{~m}$ and $63 \mathrm{~kg}$ person. A reason for this small deviation is the complex structure of the room in our scenario, making the calculation of the exact volume a difficult task. Moreover, the value from our experiment is the average $A C S$ of all 6 persons. It is thus expected to be different from the one reported in [15] of only one human subject.

In order not to require the exact volume of the room, the parameters $A C S$ and $A_{0}$ used for estimation based on (2) and (3) can be replaced by the scaled versions $A C S / V$ and $A_{0} / V$, respectively. This requires a measurement-based calibration of these ratios to be able to estimate the number of people from the RT with optimal performance. To that end, the measurement data are randomly split into two sets: a calibration set of $20 \%$ of the data for calculating the $A C S / V$

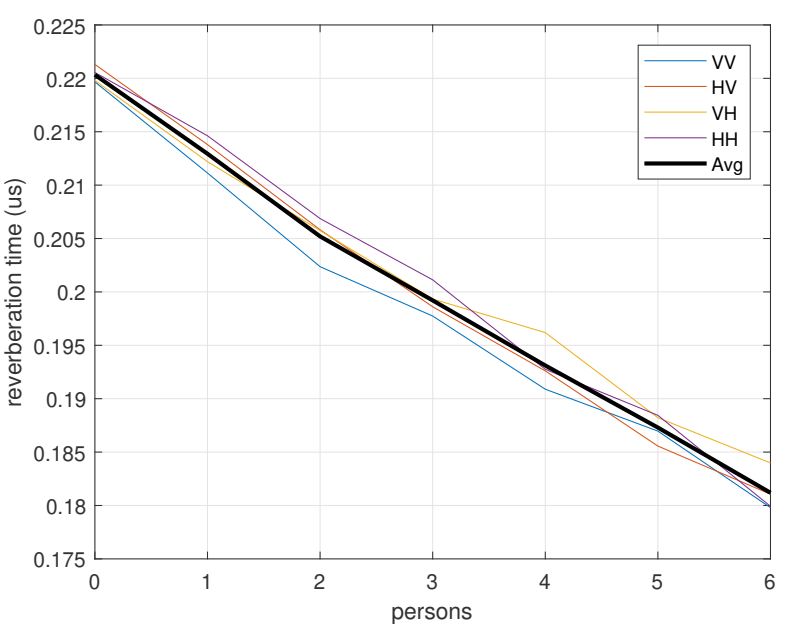

Fig. 6. RT versus the number of people inside the room for different polarizations

TABLE II

PARAMETERS CALIBRATION IN $\left(10^{-4} / \mathrm{M}\right)$

\begin{tabular}{ccc}
\hline & Full data & Calibration set $(20 \%)$ \\
\hline$A C S / V$ & 22.17 & 21 \\
$A_{0} / V$ & 607.33 & 609.5 \\
\hline
\end{tabular}

and $A_{0} / V$, and a testing set of $80 \%$ to actually estimate the number of people based on the calibrated values. Table II summaries the calculated calibration values from both the full data set and the calibration set of the channel sounding. The small difference between the values of the two sets indicates how well the estimation performance is expected to be.

\section{OCCUPANCY DETECTION ANALYSIS AND Discussion}

In this section, the number of people inside the room is estimated. The performance is measured in terms of the estimation error $e$ defined as the absolute difference between the estimate and the actual number of people

$$
e=|n-\widehat{n}|
$$

\section{A. Estimation Results via Channel Sounding}

Table II lists the calibrated ratios to be used for estimation, while $A_{n} / V$ is calculated based on the measured RT from (2) for each case. From (3), the estimate number of people is obtained as

$$
\widehat{n}=\left[\frac{A_{n} / V-A_{0} / V}{A C S / V}\right]
$$

where a simple round operator [.] is used to get the integer estimate. The estimation performance depends on the RT calculation as aforementioned. For a more accurate RT calculation, averaging of PDPs from several spatial links is used beforehand. Since the antenna elements capture uncorrelated CIRs of the same environment, averaging PDPs of the same number of people will smooth the decaying tail. This results in a more accurate regression line for the RT calculation. 


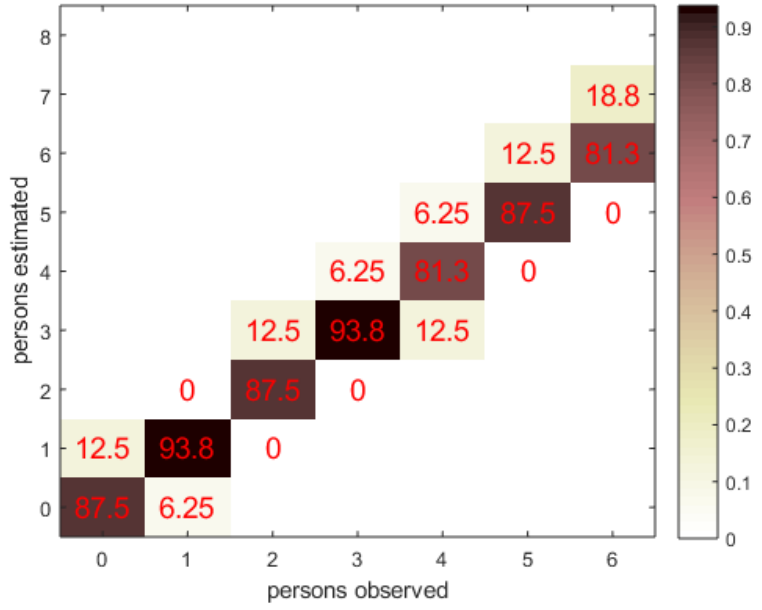

Fig. 7. Confusion matrix for the estimation percentage of the number of people in the room with spatial averaging $m=16$ via channel sounding

Fig. 8 presents the estimation error histogram for $m$ number of averaged PDPs from the testing set. For only a single spatial link $(m=1)$, the estimation error can reach up to 6 persons with an estimation success rate of only $21.4 \%$. As $m$ increases, the estimation performance improves in terms of higher success rate and smaller number of persons as estimation error. With $m=16$, the success rate is $88 \%$ with only a 1-person error of $12 \%$. Fig. 7 shows the confusion matrix for $m=16$. It gives the details of the estimation percentage for each case of the observed number of people. This clearly shows the good performance of the estimation algorithm, where all the cases have a success rate above $81 \%$, and all the estimation error larger than 1-person is $0 \%$. It is worth noting that while the persons inside the room may not be identically exposed to the propagating waves, nor having the same physical surface area, this method assumes they are. This is clear from (3), which directly influences the estimation performance. By increasing the number of antennas and the spatial averaging size, the accuracy of the RT calculation, and hence, the overall estimation performance can be further improved.

\section{B. Estimation Results via OTS UWB}

The MIMOSA channel sounder is a dedicated device for measuring the CIR with high accuracy and precision. In order to test the feasibility of our method, we use OTS products of a radio access technology that is part of available communication networks. UWB nodes, usually deployed for RTLS and communication networks, are used to measure the CIRs in the same scenario. Fig. 2 (right) shows the UWB nodes array used to have the same $8 \times 8$ MIMO setup. The $\mathrm{Rx}$ and Tx arrays are placed at the same locations as those of the channel sounder. The 6 people are again introduced into the room one by one with the difference that they are allowed to move freely while capturing the CIRs. Hence, the 200 CIRs measured per number or people can be considered uncorrelated, and consequently, PDPs averaging over time can

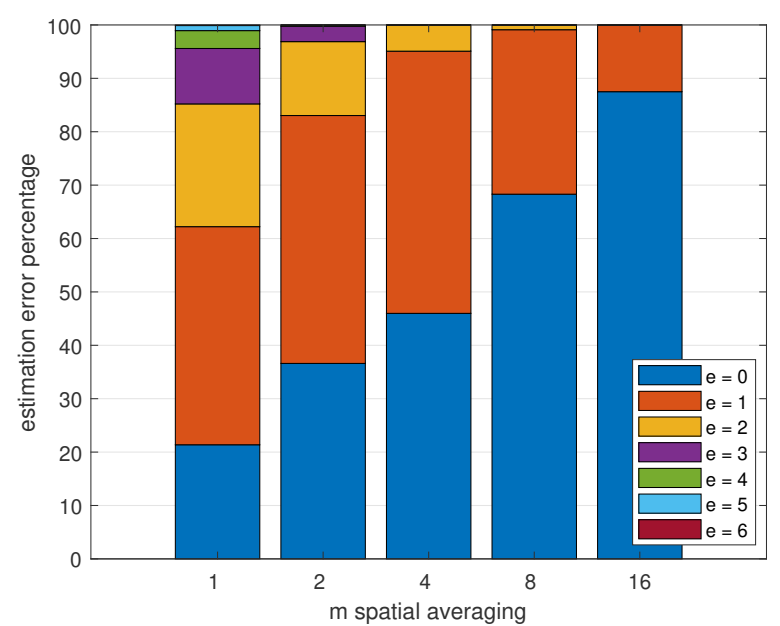

Fig. 8. Estimation error ( $\mathrm{e}=0-6$ persons $)$ histogram of the number of people in the room for different spatial averaging sizes via channel sounding

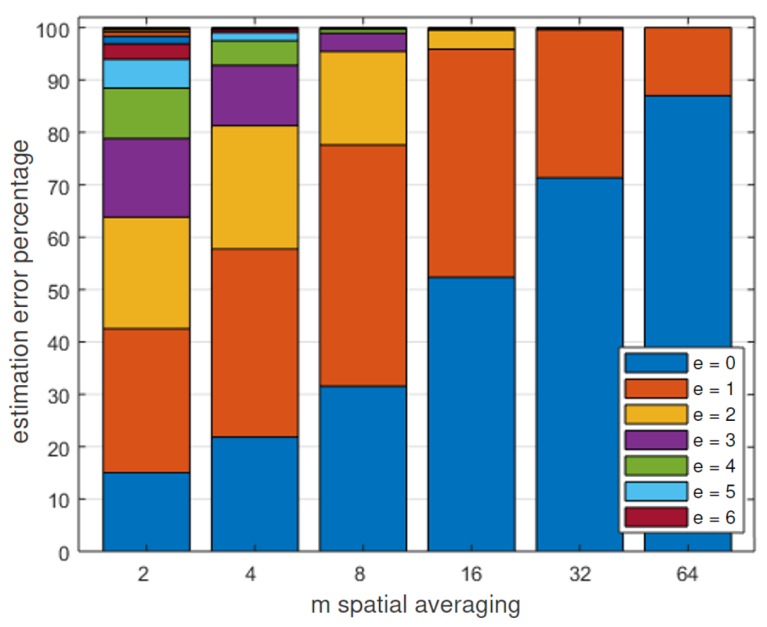

Fig. 9. Estimation error ( $\mathrm{e}=0-6$ persons) histogram of the number of people in the room for different spatial averaging sizes $m$ via UWB

enhance the accuracy of RT extraction, as an addition to spatial averaging.

Another difference that impacts the RT extraction is the characteristics of the CIRs. MIMOSA captures around 10 us of delay range with $12.5 \mathrm{~ns}$ resolution, of which only the first 5 us are plotted in Fig. 4. The used UWB chip records around 1 us of delay range starting from the LOS, with 1 ns resolution. Fig. 4 shows that the PDP in our scenario spans almost 2.5 us from the LOS. Thus, only the first $40 \%$ of the PDP following the LOS is used for calculating the regression line in the case of UWB. This is expected to degrade the accuracy of the RT calculation. Fig. 9 shows the estimation error histogram for different sizes of only spatial averaging. Although a larger averaging size results in a higher success rate, the performance is lower compared to the channel sounder. With $m=16$, the success rate is about 53\% with up to 3-person errors in the case of UWB. In order to reach the channel sounding performance of $88 \%$ success rate and only 1-person error, the 


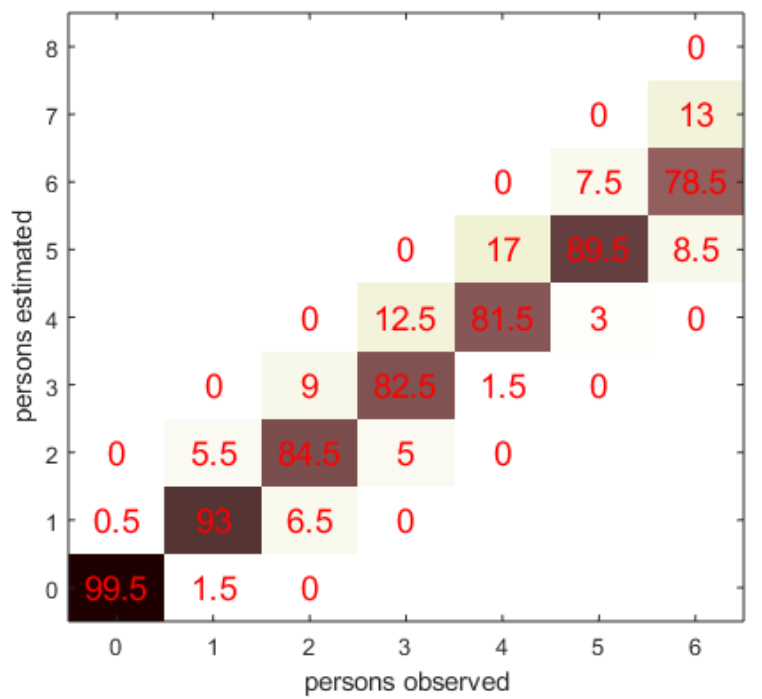

Fig. 10. Confusion matrix for the estimation percentage of the number of people in the room with spatial averaging $m=64$ via UWB

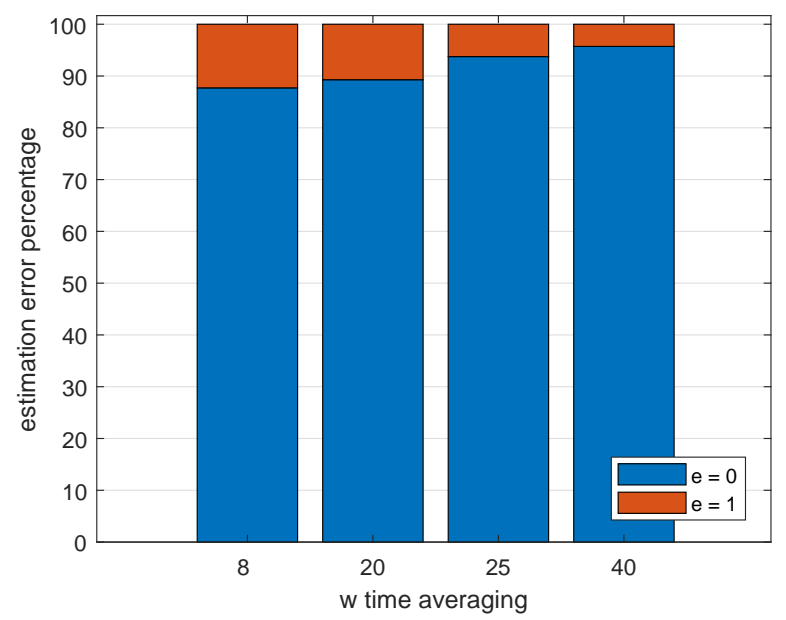

Fig. 11. Estimation error ( $\mathrm{e}=0-1$ person) histogram of the number of people in the room for spatial averaging $m=32$ and different time averaging sizes $w$ via UWB

spatial averaging size should increase to 64, as shown in Fig. 9. The confusion matrix for the estimation percentage with $m=64$ is shown in Fig. 10. It again shows the good performance of the algorithm using UWB, where all the errors above 1-person are $0 \%$.

In addition to spatial averaging, time averaging of PDPs can be used to enhance the estimation performance as aforementioned. Fig. 11 shows the estimation error histogram for spatial averaging size $m=32$ and different time averaging sizes $w$. With only $w=8$, the same performance of the 64 spatial averaging can be achieved with less number of antennas $(m=32)$. Increasing the time averaging size results in higher success rate, reaching $95 \%$ with $w=40$ as shown in Fig. 11. While it is easier to reach higher time averaging size compared to having more antennas for spatial averaging, the performance enhancement of time averaging highly depends

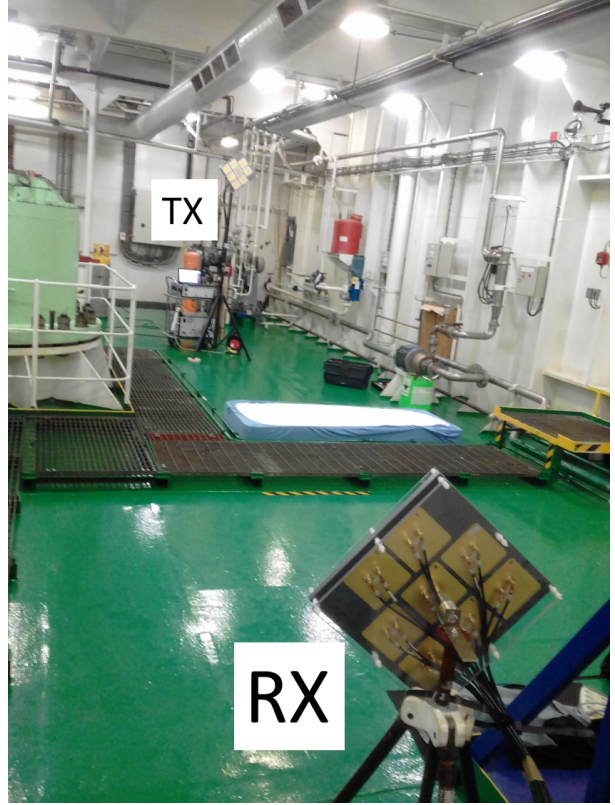

Fig. 12. Measurement setup for fall detection via MIMOSA channel sounder

on the scenario. In a stationary environment, where movement is limited, having uncorrelated CIRs over time is difficult. Thus, spatial averaging is considered more robust compared to time averaging, even though it requires more antennas.

\section{FAll Detection in ReVERberant EnVIRONMENTS}

Knowledge of the location of people on ships has a wide range of applications in commercial solutions and during the duty of state officials. In the previous section, we showed that the number of people inside harsh reverberant environments like ship compartments can be accurately estimated using CIRs available in many communication systems. A highly critical situation is when a crew member working alone in isolated areas falls on the ground. Therefore, we investigate the detection of a fall event in such environments using the same measured CIRs. Most radar-based fall detection studies focus on residential environments for monitoring elderly people in their daily activities and promptly detect fall events, enabling timely and effective intervention [18]. In these environments, it is much easier to extract information from the phase or Doppler domain for velocity or micro-Doppler signature analysis [18]. An example of how the environment can impact the detection performance can be found in [20], where the fall detection precision of a one-class support-vector machine classifier drops from $96 \%$ in an anechoic chamber, to $83 \%$ in a dormitory room. In this section, we investigate the possibility of detecting a fall from a standing position in a reverberant environment using CIRs of communication systems, which are measured at a much lower rate compared to radar sensors.

\section{A. Measurement and Processing}

MIMOSA channel sounder is used in the same below-deck chamber as shown in Fig. 12. The Tx is fixed to face the $\mathrm{Rx}$ in a LOS condition, and a mattress is located in the 


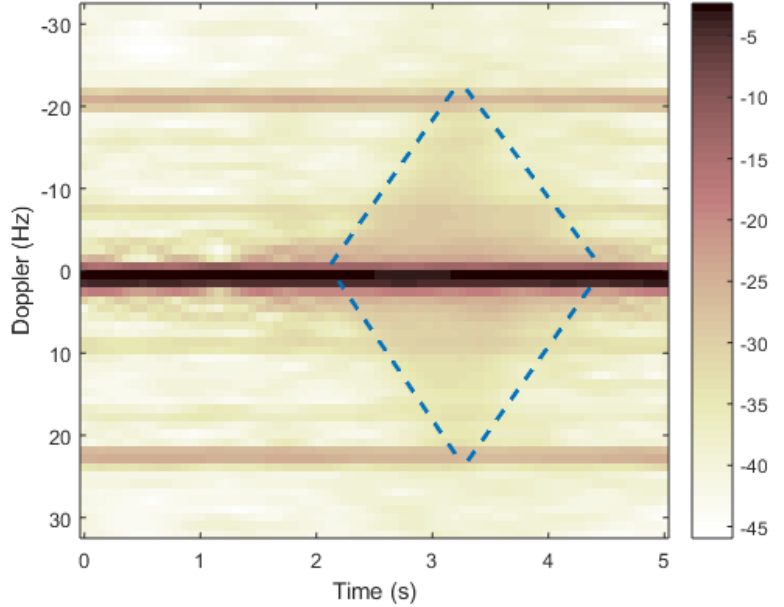

Fig. 13. Time evolution of Doppler-power profiles in $\mathrm{dB}$ for a fall event. The blue region indicates the Doppler components resulting from the fall

middle so that the fall is perpendicular to the LOS. This represents a worst-case scenario since the fall movement is in the plane orthogonal to the LOS direction, reducing the effective Doppler shift. The impact of the fall orientation has been studied in many papers. Doppler signature is sensitive to the direction of motion defined by the aspect angle, which is the angle between target motion trajectory and the radial LOS path between the radar and the target. The most distinctive signatures appear when target is moving towards or away from the radar, leading to the maximum Doppler spread. As the aspect angle increases, the Doppler effect also decreases. When the aspect angle approaches $90^{\circ}$, Doppler signal becomes strongly attenuated. It is reported that the fall detection performance can drop approximately to below $50 \%$ for target directions with angles close to $90^{\circ}$ [25]. This is in case of non-reverberating environments, where diffuse scattering is not dominant and the Doppler paths can be distinguished.

The experiment consists of a person walking, sitting (squatting down) and falling on the mattress. Each activity is repeated 3 times of $5 \mathrm{~s}$ measurement duration. Eight dualpolarized antenna elements at the $\mathrm{Tx}$ and one at the $\mathrm{Rx}$ are used to measure the CIRs while the person is doing the activity, thus making use of the parallel transmission to have a larger sample size. Motion in the environment can be detected using the Doppler-power profile (DPP); faster movements result in larger Doppler spreads. A person can fall to the ground at $4 \mathrm{~m} / \mathrm{s}$, with the head impact velocity exceeding $6 \mathrm{~m} / \mathrm{s}$ from standing [26]. This corresponds to around $18 \mathrm{~Hz}$ of Doppler shift. Thus, CIRs are captured at a $64 \mathrm{~Hz}$ rate, providing Doppler frequencies up to $32 \mathrm{~Hz}$, according to the Nyquist theorem. Each DPP is calculated from 64 consecutive CIRs averaged over the whole bandwidth per polarization per antenna. Fig. 13 shows the time evolution of DPPs in dB, over the $5 \mathrm{~s}$ duration of one fall activity. It shows the constant LOS component with zero Doppler since both Tx and Rx are fixed. The components representing the fall are in the area marked by the blue lines, which are below $20 \mathrm{~Hz}$ as expected. Two constant components at $+/-21 \mathrm{~Hz}$ can be seen, probably due

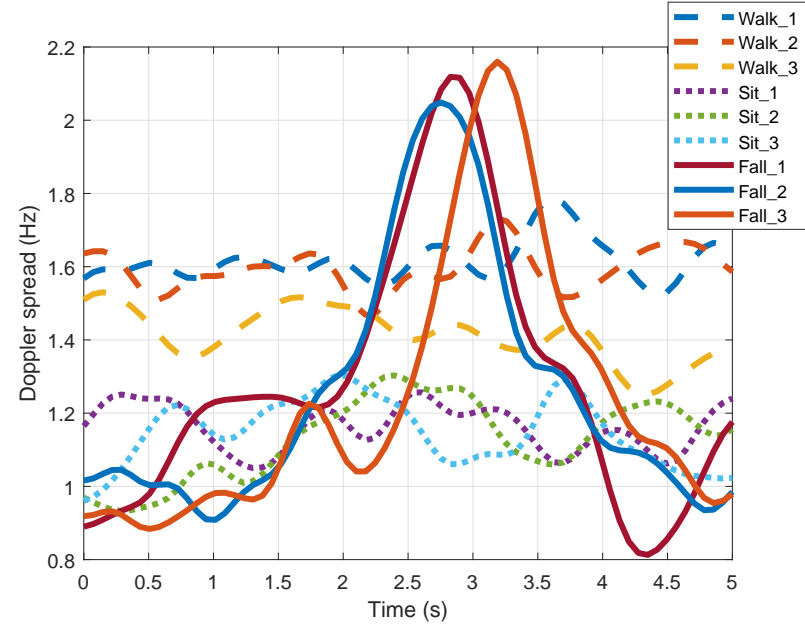

Fig. 14. RMS Doppler spread for fall, walk and sit activities, each performed 3 times

to the vibration caused by the ship's engine.

Fig. 14 shows the RMS Doppler spread calculated from the DPP over the $5 \mathrm{~s}$ duration. DPPs per event are first averaged over all antennas and polarizations to have 3 records per activity. To calculate the RMS Doppler spread, the DPPs are clipped to remove components above $20 \mathrm{~Hz}$ unrelated to the fall event. A fall can be detected when a peak in the Doppler spread is captured, which marks the increasing and then decreasing velocity of the fall. This differentiates the fall from other activities like walking and sitting. Fig. 14 clearly shows that the Doppler spread for the fall has higher peak values compared to the other normal activities.

\section{B. Feature Extraction and Detection Results}

After verifying that a fall can be differentiated by the Doppler spread pattern, a classifier is needed to detect a fall event based on certain features. We choose a Naive Bayes classifier with only one feature for simplicity [27]. The feature is extracted from each Doppler spread pattern of $5 \mathrm{~s}$ duration. These patterns are calculated from the DPP per antenna, per polarization, and per event resulting in a total of 48 patterns per class of activity. Several features are investigated, e.g., the mean, peak, peak-to-mean ratio, maximum gradient, and variance of the Doppler spread patterns. It is found that the variance of the Doppler spread over the $5 \mathrm{~s}$ duration gives the best separation among the 3 classes, namely fall, walk and sit shown in Fig. 15.

The sample data are split into a training set for the classifier model development and a testing set for the model validation. Since the data size is limited to 48 samples per class, the k-fold cross-validation method is used with $\mathrm{k}=3$. The data are split into 3 folds where each fold is used as the testing set while the others as the training set. Finally, the classification results are averaged over the 3 splits. Even though we have 3 classes, our main aim is to detect only the fall event. Thus, performance metrics for binary classification (positive/negative) are used. These include detection accuracy (ratio of true to total predictions), precision (ratio of true positive to total positive 


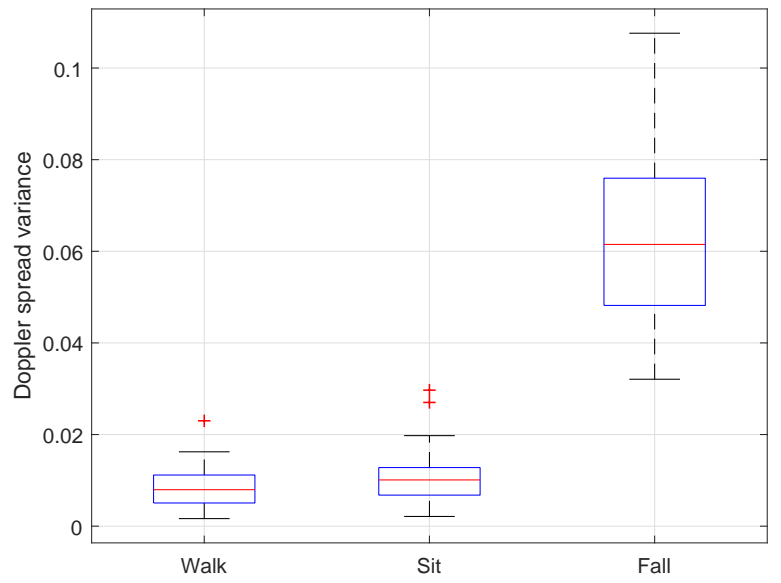

Fig. 15. Doppler spread variance over the $5 \mathrm{~s}$ duration for each class of activity, used as a classification feature with good separation

TABLE III

Fall Detection Performance Metrics

\begin{tabular}{ccc}
\hline Training set per class & 32 samples (66\%) & 8 samples (17\%) \\
\hline Accuracy & $98.61 \%$ & $97.92 \%$ \\
Precision & $96 \%$ & $94.12 \%$ \\
Sensitivity & $100 \%$ & $100 \%$ \\
\hline
\end{tabular}

predictions) and sensitivity (ratio of true positive predictions to total positive observations). Fig. 16 shows the confusion matrix for the class predictions as percentage of the observations, averaged for $\mathrm{k}=3$ folds. It shows that $100 \%$ of the fall events are detected, and only $4.17 \%$ of the sit events are mispredicted as falling. It also shows that this classifier can not differentiate between sit and walk events, since $62.5 \%$ of the sit events are mispredicted as walking.

Table III lists the performance metrics for the fall detection. With $\mathrm{k}=3$ folds, one third of the data are used for validation, while 66\% (32 samples per class) are for training. This gives a very good performance as the metrics consisting of accuracy, precision and sensitivity are all above $96 \%$. The table also includes performance metrics when only $17 \%$ of the data $(8$ samples per class) are used for training. While the sensitivity is still $100 \%$, a small degradation in accuracy and precision can be noticed. Nevertheless, all metrics are above $94 \%$, which is a good performance for a single feature classifier with only 8 training samples per class.

\section{CONCLUSION}

Industrial environments are characterized by high time and angular dispersion due to scattering from complex metallic structures. Such harsh environments experience reverberation behaviour similar to cavities. Based on the theory of Room Electromagnetics, this paper explores the feasibility of estimating the number of people inside a reverberant ship compartment by means of only measuring the reverberation time. We observe that the reverberation time is the same,

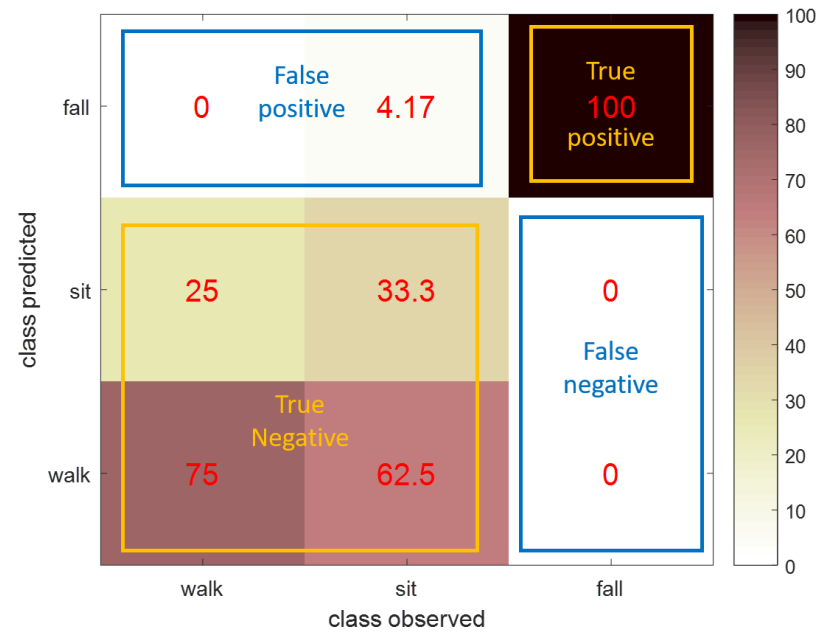

Fig. 16. Confusion matrix for the classification of the testing set with $\mathrm{k}=3$ folds, with prediction types indicated

independent of the antenna or the location used for measurement inside the room. Our findings verify that there is an inverse relation between the number of people inside the room and the reverberation time. More people absorb more energy, decreasing the reverberation time. A calibration of the absorption parameters of the empty room and the average human body is needed before performing the estimation, which is done via measurements. While the estimation performance is very low in case of a single antenna, it can be enhanced via spatial averaging from multiple antennas. In addition, time-averaging can be used to further enhance the estimation performance when the measured channel is non-stationary due to the movement of people. The estimation algorithm depends on the channel impulse response (CIR), a metric that can be found in wideband communication systems. With OTS UWB nodes that are originally used for localization and communication, we estimated the number of people ranging from 0 to 6 persons with a success rate of $95 \%$ and only 1-person error. Moreover, the CIRs can be used to detect when a person alone has fallen to the ground via Doppler analysis. Doppler frequencies up to $20 \mathrm{~Hz}$ are used, so the radio channel can be sampled at only $40 \mathrm{~Hz}$ rate. While most studies on fall detection use micro-Doppler signatures extracted with sampling rates above $1 \mathrm{kHz}$, they only focus on residential environments where such signatures can be easily detected. In a reverberant scenario, we found that the RMS Doppler spread has a peak that differentiates a fall from sitting or walking. A simple Bayes classifier is used for fall detection, with the variance of the Doppler spread as its feature. Using 3-fold cross-validation, the fall is detected with $98.6 \%$ accuracy, $96 \%$ precision, and $100 \%$ sensitivity. Future work will include testing the developed algorithms in different scenarios to characterize the performance dependency on the environment as well as the number of people.

\section{REFERENCES}

[1] G. B. Tait and M. B. Slocum, "Electromagnetic environment characterization of below-deck spaces in ships," in 2008 IEEE International Symposium on Electromagnetic Compatibility. IEEE, 2008, pp. 1-6. 
[2] G. Tait, M. Slocum, and R. Richardson, "On multipath propagation in electrically large reflective spaces," IEEE Antennas and Wireless Propagation Letters, vol. 8, pp. 232-235, 2009.

[3] A. Mariscotti, M. Sassi, A. Qualizza, and M. Lenardon, "On the propagation of wireless signals on board ships," in 2010 IEEE Instrumentation \& Measurement Technology Conference Proceedings. IEEE, 2010, pp. $1418-1423$.

[4] K. Wanuga, R. Measel, C. S. Lester, D. J. Bucci, D. Gonzalez, R. Primerano, M. Kam, and K. R. Dandekar, "Performance evaluation of MIMO OFDM systems in on-ship below-deck environments," IEEE Antennas and Wireless Propagation Letters, vol. 13, pp. 173-176, 2014.

[5] R. Measel, C. S. Lester, D. J. Bucci, K. Wanuga, G. Tait, R. Primerano, K. R. Dandekar, and M. Kam, "An empirical study on the performance of wireless OFDM communications in highly reverberant environments," IEEE Transactions on Wireless Communications, vol. 15, no. 7, pp. $4802-4812,2016$

[6] S. T. Kouyoumdjieva, P. Danielis, and G. Karlsson, "Survey of nonimage based approaches for counting people," IEEE Communications Surveys Tutorials, pp. 1-1, 2019.

[7] M.-G. Di Benedetto, UWB communication systems: a comprehensive overview. Hindawi Publishing Corporation, 2006, vol. 5.

[8] J. W. Choi, D. H. Yim, and S. H. Cho, "People counting based on an IR-UWB radar sensor," IEEE Sensors Journal, vol. 17, no. 17, pp. 5717-5727, 2017

[9] J. Poutanen, J. Salmi, K. Haneda, V. Kolmonen, and P. Vainikainen, "Angular and shadowing characteristics of dense multipath components in indoor radio channels," IEEE Transactions on Antennas and Propagation, vol. 59, no. 1, pp. 245-253, 2011.

10] H. Kuttruff, Room acoustics. Crc Press, 2016.

[11] J. B. Andersen, J. Nielsen, G. Pedersen, G. Bauch, and J. Herdin, "Room electromagnetics," IEEE Antennas and Propagation Magazine, vol. 49 no. 2, pp. 27-33, 2007.

[12] G. Steinböck, T. Pedersen, B. H. Fleury, W. Wang, and R. Raulefs, "Experimental validation of the reverberation effect in room electromagnetics," IEEE Transactions on Antennas and Propagation, vol. 63 , no. 5, pp. 2041-2053, 2015.

[13] J. B. Andersen, K. L. Chee, M. Jacob, G. F. Pedersen, and T. Kurner, "Reverberation and absorption in an aircraft cabin with the impact of passengers," IEEE Transactions on Antennas and Propagation, vol. 60 no. 5, pp. 2472-2480, 2012.

[14] A. Bamba, W. Joseph, J. B. Andersen, E. Tanghe, G. Vermeeren, D. Plets, J. Ø. Nielsen, and L. Martens, "Experimental assessment of specific absorption rate using room electromagnetics," IEEE Transactions on Electromagnetic Compatibility, vol. 54, no. 4, pp. 747-757, 2012.

[15] A. Bamba, D. P. Gaillot, E. Tanghe, G. Vermeeren, W. Joseph, M. Lienard, and L. Martens, "Assessing whole-body absorption cross section for diffuse exposure from reverberation chamber measurements," IEEE Transactions on Electromagnetic Compatibility, vol. 57, no. 1, pp. $27-$ 34, 2015.

[16] F. Bellens, F. Quitin, F. Horlin, and P. De Doncker, "UWB channel analysis within a moving car," in 2009 9th International Conference on Intelligent Transport Systems Telecommunications, (ITST), 2009, pp 681-684.

[17] S. Adireddy, Lang Tong, and H. Viswanathan, "Optimal placement of training for frequency-selective block-fading channels," IEEE Transactions on Information Theory, vol. 48, no. 8, pp. 2338-2353, 2002.

[18] E. Cippitelli, F. Fioranelli, E. Gambi, and S. Spinsante, "Radar and RGB-depth sensors for fall detection: A review," IEEE Sensors Journal, vol. 17, no. 12, pp. 3585-3604, 2017.

[19] H. Wang, D. Zhang, Y. Wang, J. Ma, Y. Wang, and S. Li, "RT-fall: A real-time and contactless fall detection system with commodity WiFi devices," IEEE Transactions on Mobile Computing, vol. 16, no. 2, pp. 511-526, 2017.

[20] Y. Wang, K. Wu, and L. M. Ni, "Wifall: Device-free fall detection by wireless networks," IEEE Transactions on Mobile Computing, vol. 16 , no. 2, pp. 581-594, 2017.

[21] P. Laly, D. P. Gaillot, M. Liénard, P. Degauque, E. Tanghe, W. Joseph, and L. Martens, "Flexible real-time MIMO channel sounder for multidimensional polarimetric parameter estimation," in Antenna Measurements \& Applications (CAMA), 2015 IEEE Conference on. IEEE, 2015, pp. $1-3$.

[22] B. Van Herbruggen, B. Jooris, J. Rossey, M. Ridolfi, N. Macoir, Q. Van den Brande, S. Lemey, and E. De Poorter, "Wi-pos: A lowcost, open source ultra-wideband (uwb) hardware platform with long range sub-ghz backbone," Sensors, vol. 19, no. 7, 2019.
[23] “Application notes," Decawave Ltd., Tech. Rep. [Online]. Available: https://www.decawave.com/application-notes/

[24] A. Kalyanaraman, Y. Zeng, S. Rakshit, and V. Jain, "Caraokey : Car states sensing via the ultra-wideband keyless infrastructure," in 2020 17th Annual IEEE International Conference on Sensing, Communication, and Networking (SECON), 2020, pp. 1-9.

[25] B. Erol and M. Amin, "Effects of range spread and aspect angle on radar fall detection," in 2016 IEEE Sensor Array and Multichannel Signal Processing Workshop (SAM), 2016, pp. 1-5.

[26] M. Hajiaghamemar, M. Seidi, J. R. Ferguson, and V. Caccese, "Measurement of head impact due to standing fall in adults using anthropomorphic test dummies," Annals of biomedical engineering, vol. 43, no. 9, pp. 2143-2152, 2015.

[27] H. Zhang, "The optimality of naive bayes," Proceedings of the Seventeenth International Florida Artificial Intelligence Research Society Conference, FLAIRS, vol. 2, 012004.

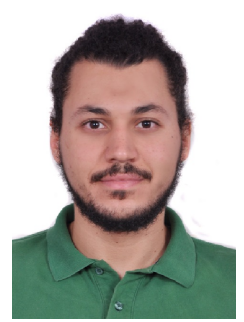

Marwan Yusuf received the M. Sc. degree in Electrical Engineering from Istanbul Medipol University (Turkey) in 2016 where he was a Research Assistant in the Communications, Signal Processing, and Networking Center (CoSiNC). His scientific work focused on physical layer security for wireless channels. Since January 2018, he has been a Doctoral Researcher in the Department of Information Technology at Ghent University, Belgium (IMECUGent/INTEC) where he works on measurementbased modelling of indoor and outdoor wireless propagation with emphasis on vehicular communication channels.

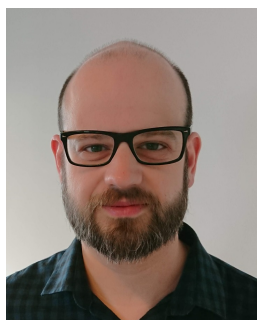

Emmeric Tanghe was born in Tielt, Belgium, on August 31, 1982. He received the M. Sc. degree in Electrical Engineering from Ghent University (Ghent, Belgium) in 2005 and the $\mathrm{Ph}$. D. degree in electrical engineering from the same institution in 2011. From September 2005 until May 2011, he was a Research Assistant with the Department of Information Technology at Ghent University (imecUGent/INTEC). His scientific work focused on the modeling of indoor and outdoor propagation through field measurements. Since May 2011, he has been a Postdoctoral Researcher with the same institution and continues his work in propagation modeling. From October 2012 until September 2018, he was a Postdoctoral Fellow of the FWO-V (Research Foundation - Flanders). In October 2015 he became a part-time professor in medical applications of electromagnetic fields in and around the human body.

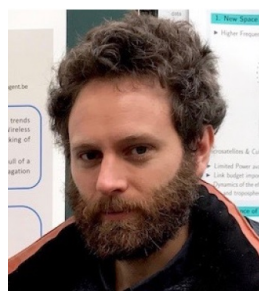

Brecht De Beelde received a M.Sc degree in Electrical Engineering from Ghent University (Belgium) in 2013. He started his career as an embedded SW engineer at the Low-Power Wireless Division of Qorvo Inc. where he worked on BLE and ZigBee communication controllers. He joined the WAVES research group of the Department of Information Technology (INTEC) of Ghent University in 2018, where he is currently working towards a $\mathrm{PhD}$ degree. His research interests are channel modelling and wireless network planning in industrial environments and at mmWave and sub-Terahertz frequencies. 


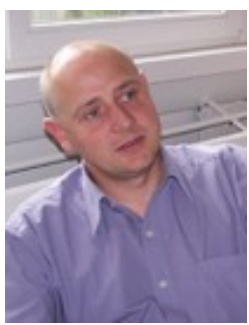

Pierre Laly is an engineer of study. His main current areas of research are the study, design and production of electronic cards to build prototypes dedicated to digital and/or analog telecommunications. He realizes the implementation and programming of microcontrollers, DSP, CPLD, FPGA components and the study and the realization of sub systems for the recovery and the statistical treatment of the information of the various prototypes.

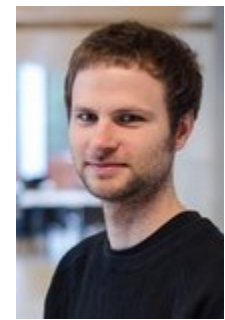

Matteo Ridolfi received the M.Sc. degree in telecommunication engineering from the University of Bologna, Italy, in 2016. He is currently a Ph.D. Researcher with the IDLab Research Group, imec, Ghent University. He is the author and coauthor of various publications on Ultra wideband localization solutions. His research focus is on indoor localization systems, mainly targeting solutions with Ultra wideband technology. His interests include localization algorithms, scalability and accuracy analysis, self-calibrating networks, and collaborative localiza-

tion.

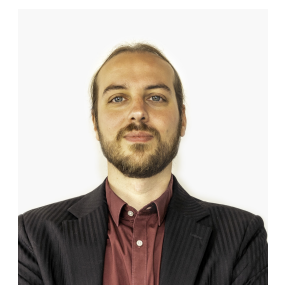

Eli De Poorter is professor at the IDLab research group from Ghent University and imec (https://idlab.technology). He received his master degree in Computer Science Engineering from Ghent University, Belgium, in 2006, his Ph.D. degree in 2011 at the Department of Information Technology at Ghent University and he became professor at IDLab in 2015. Since 2017, he is also affiliated with the IMEC research institute. His team performs research on wireless communication technologies such as (indoor) localization solutions, wireless IoT solutions and machine learning for wireless systems. He performs both fundamental and applied research. For his fundamental research he is currently the coordinator of several research projects (SBO, FWO, GOA, etc.) and has over 180 publications in international journals or in the proceedings of international conferences. For his applied research, he collaborates with (Flemish) industry partners to transfer research results to industrial applications as well as to solve challenging industrial research problems. Prof. De Poorter is also co-founder of the lopos spin-off company (https://lopos.be) which offers privacy-aware UWB wearables for industrial safety.

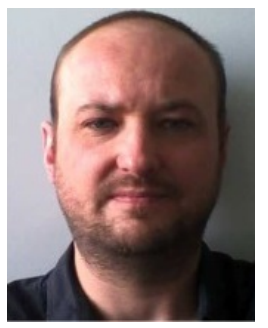

Davy P. Gaillot received the B.S. degree in mechanical engineering from Ecole Nationale dIngnieurs de Metz (ENIM), Metz, France, in 2002, and the masters degree in mechanics, materials, structures, and processes from the University of Metz, Metz, France. He also received the Ph.D. degree, in 2007, from the Department of Materials Science and Engineering at the Georgia Institute of Technology, Atlanta, GA, USA. He was a Postdoctoral Fellow at the Institute of Electronics, Microelectronics, and Nanotechnology (IEMN) in Villeneuve dAscq, France. Since 2008, he has been an Associate Professor at the University of Lille 1 in the IEMNTELICE Group and recently also joined the Institute of Research in Software and Hardware Components for Information and Advanced Communications (IRCICA). His research activities include radiocommunications, propagation, channel modeling and experimental channel sounding from the wireless telecommunications frequency range to $\mathrm{THz}$. $\mathrm{He}$ currently is one of the French substitute delegates and active participant for the European COST action CA15105 IRACON (Incluse Radio Communications Networks for $5 \mathrm{G}$ and Beyond).

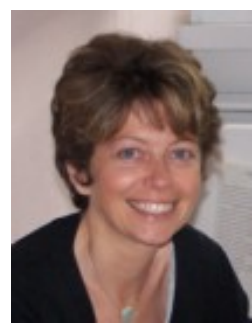

Martine Lienard received the Ph.D. degree from the University of Lille, Lille, France, in 1993. Since 1990, she has been with the Telecommunications, Interferences and Electromagnetic Compatibility (TELICE) group of the Institute of Electronic, Microelectronic and Nanotechnology (IEMN) of University of Lille. She is currently a Professor at University of Lille and Head of the TELICE Group. Her current research deals with mobile localization techniques in non cooperative mode and theoretical and experimental channel characterization in complex environment. She is also involved in challenges of MIMO and Massive MIMO techniques, mainly dedicated for communication in rail and road transportation systems and industry 4.0.

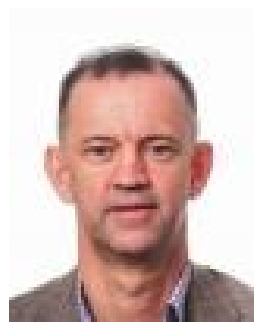

Luc Martens received the M.Sc. degree in electrical engineering from the Ghent University, Belgium in July 1986. From September 1986 to December 1990 he was a research assistant at the Department of Information Technology (INTEC) of the same university. During this period, his scientific work was focused on the physical aspects of hyperthermic cancer therapy. His research work dealt with electromagnetic and thermal modelling and with the development of measurement systems for that application. This work led to the Ph.D. degree in December 1990. Since 1991, he manages the WAVES research group at INTEC. This group is since 2004 part of the iMinds institute and since April 1993 he is Professor at Ghent University. His experience and current interests are in modelling and measurement of electromagnetic channels, of electromagnetic exposure e.g. around telecommunication networks and systems such as cellular base station antennas, and of energy consumption in wireless networks. He is author or co-author of more than 300 publications in the domain of electromagnetic channel predictions, dosimetry, exposure systems and health and wireless communications.

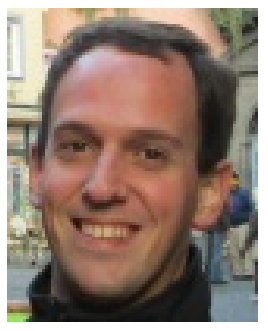

Wout Joseph was born in Ostend, Belgium on October 21, 1977. He received the M. Sc. degree in electrical engineering from Ghent University (Belgium), in July 2000. From September 2000 to March 2005 he was a research assistant at the Department of Information Technology (INTEC) of the same university. During this period, his scientific work was focused on electromagnetic exposure assessment. His research work dealt with measuring and modelling of electromagnetic fields around base stations for mobile communications related to the health effects of the exposure to electromagnetic radiation. This work led to a Ph. D. degree in March 2005. From April 2005-2009, he was postdoctoral researcher for iMinds-UGent/INTEC. From October 2007 to October 2013, he was a Post-Doctoral Fellow of the FWO-V (Research Foundation Flanders). Since October 2009, he is professor in the domain of Experimental Characterization of wireless communication systems. He is IMEC PI since 2017. His professional interests are electromagnetic field exposure assessment, in-body electromagnetic field modelling, electromagnetic medical applications, propagation for wireless communication systems, IoT, antennas and calibration. Furthermore, he specializes in wireless performance analysis and Quality of Experience. 